\title{
Propuesta de Tasa Retributiva por Emisiones de Contaminantes Atmosféricos Provenientes de Fuentes Fijas Ubicadas en Bogotá D.C.
}

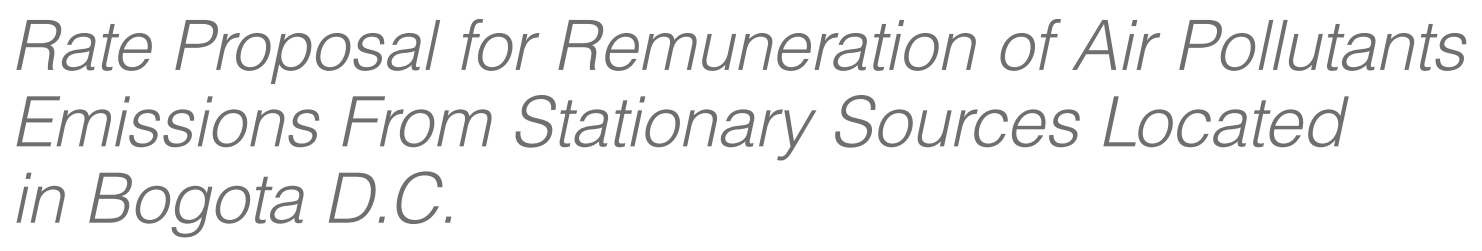

Herrera Torres Gabriel', Alvis Colmenares María F. ${ }^{2}$, Ayala Mora Diana C. ${ }^{3}$

\author{
${ }^{1}$ Facultad de Ingeniería, Programa de Ingeniería Ambiental y Sanitaria, Universidad de La Salle, Bogotá. Colombia, \\ gaherrera@lasalle.edu.co \\ ${ }^{2}$ Facultad de Ingeniería, Programa de Ingeniería Ambiental y Sanitaria, Universidad de La Salle, Bogotá. Colombia, \\ maffee10@hotmail.com \\ ${ }^{3}$ Facultad de Ingeniería, Programa de Ingeniería Ambiental y Sanitaria, Universidad de La Salle, Bogotá. Colombia, \\ nanis1009@hotmail.com
}

\section{Resumen}

Este articulo presenta la propuesta metodológica para el establecimiento de la tasa retributiva por la utilización directa de la atmósfera como receptor de emisiones contaminantes provenientes de fuentes fijas puntuales en la cuidad de Bogotá D. C. Inicialmente, con base en el inventario de emisiones de fuentes fijas y con el análisis de la calidad del aire, se identificaron los contaminantes que emiten las industrias y los contaminantes que son regulados por la red de monitoreo de la calidad de aire de Bogotá (RMCAB). Se seleccionó el material particulado $\left(\mathrm{PM}_{10}\right)$, los Óxidos de Azufre $\left(\mathrm{SO}_{\mathrm{x}}\right)$ y los Óxidos de Nitrógeno $\left(\mathrm{NO}_{\mathrm{x}}\right)$ como los contaminantes atmosféricos que deben ser objeto de cobro de la tasa retributiva. Además de la selección de los contaminantes objetos de cobro se realizó el análisis de la estructura de la tasa retributiva la cual se centró en la descripción de cuatro elementos claves; del hecho generador, la base imponible, el sujeto pasivo y el activo de la tasa. Teniendo en cuenta los costos sociales los cuales están relacionados con la inversión que realiza el Distrito para la atención a pacientes que presentan enfermedades respiratorias agudas ERA's asociadas a la contaminación atmosférica en Bogotá; y los costos del programa de control y vigilancia de la calidad del aire de Bogotá. Se definieron las tarifas para el cobro de la tasa retributiva en $2810 \$ / \mathrm{Kg}$ para el $\mathrm{PM}_{10}, 2816 \$ / \mathrm{Kg}$ para los SOx, y $2866 \$ / \mathrm{Kg}$ para los NOx. Finalmente, se estableció el modelo de cobro de la tasa, el cual será el resultado de multiplicar la tarifa correspondiente para cada uno de los contaminantes objetos de cobro, expresada en $(\$ / \mathrm{Kg})$, por la carga contaminante emitida por la fuente expresada en (Kg/día) por el número total de días de operación de la fuente de emisión al año.

Palabras clave: contaminación atmosférica, instrumento económico, tasas retributivas.

\section{Abstract}

The objective of this project is to develop a methodological proposal for the establishment of the retributive rate for the direct use of the atmosphere as the receptor of pollutant emissions that come from stationary resources on Bogotá D.C. By means of the emissions from stationary sources inventory and the air quality analysis, the pollutant that are emitted by the industries and the ones that are regulated by the network observations of the were identified selecting the particulated matter (PM10), sulfur oxides (SOx), and nitrogen oxides (NOx) as the atmospheric pollutants that should be the object of payment in the retributive rate. Besides the selection of the pollutants that should be in the payment, the analysis of the retributive rate structure was made witch was based on the description or four key elements the generated 
fact, the tax base, the passive subject, and the fee of the rate. taking into account the social costs which are related to the investment being made by the district for the treatment of patients that present acute respiratory diseases ERA's, associated and the costs of program control and monitoring of the air quality in Bogotá, the tariffs of the payment of the retributive rate were redefined in $281 \$ / \mathrm{Kg}$ for the $\mathrm{PM}_{10}, 2816$ $\$ / \mathrm{kg}$ for the $\mathrm{SO}_{\mathrm{x}}$ and $2866 \$ / \mathrm{kg}$ for $\mathrm{NO}_{\mathrm{x}}$. Finally a new model of the payment was established, which is the result of the multiplication of the respective tariff for each of the pollutants that were selected as object of payment, expressed in $(\$ / \mathrm{kg})$ times, the charge of the pollutants emitted by the source expressed in $(\mathrm{kg} /$ day).times the total number of days of the operation of the source emissions in a year.

Keywords: air pollution, economic instrument, rate retributive.

\section{Introducción}

La contaminación del aire en los centros urbanos es uno de los problemas de mayor preocupación para los colombianos y el generador de los mayores costos sociales después de la contaminación del agua y de los desastres naturales. Estos costos han sido estimados en 1,5 billones de pesos anuales, y están relacionados con efectos adversos sobre la salud de la población, con la mortalidad y morbilidad asociadas a enfermedades respiratorias y cardiovasculares. Las causas que originan esta contaminación son diversas, pero el mayor índice es provocado por las actividades industriales y comerciales que aportan al aire emisión de gases, vapores y humos contaminantes, lo que deteriora de esta forma la calidad del aire.

Teniendo en cuenta el problema, se hace necesario el desarrollo de políticas sociales, ambientales y económicas que incentiven un cambio en el comportamiento de los agentes generadores de la contaminación atmosférica. Es por esto que desde 1974, con el Código Nacional de Recursos Naturales, (Decreto-Ley 2811 de 1974), se creó la tasa retributiva como instrumento económico. La tasa fue definida como el costo que cobra el estado por permitir la utilización del medio ambiente como receptor de residuos y emisiones; el establecimiento de la tasa busca cambiar el comportamiento del contaminador por lo que permitirá que sea él quien decida la forma más eficiente y menos costosa de reducir la contaminación que genera, en lugar de pagar la tasa. La tasa retributiva ha sido reglamentada para vertimientos de agua, pero hasta la fecha no ha sido desarrollada para la prevención y mitigación de la contaminación atmosférica.

Actualmente, la ciudad de Bogotá, a través de las políticas del Distrito para el mejoramiento de la calidad del aire en la ciudad, se encuentra adelantando programas con el objeto de reducir la contribución de las fuentes fijas puntuales a la contaminación atmosférica por material particulado. Para ello, se han establecido medidas estructurales que contemplan la actualización del inventario de emisiones, la clasificación de la ciudad en áreas fuente de contaminación, la caracterización fisicoquímica del material particulado, la formulación del plan decenal de mejoramiento de la calidad del aire de Bogotá D.C., y la inspección, vigilancia y control a fuentes fijas de emisión para el cumplimiento de la Resolución 1208 del 2003, entre otras medidas.

Con la imposición de una tasa retributiva en materia de aire no solo se quiere lograr una disminución de la contaminación atmosférica, sino que a la vez se pretende incentivar a los industriales hacia una reconversión en tecnologías más limpias. Este instrumento económico hace parte de la Política Nacional para la producción más limpia que busca cambiar el paradigma del control de la contaminación al final del tubo a un concepto moderno y costo efectivo de prevención y minimización de los residuos, mediante la promoción de inversiones en equipos y procesos más limpios.

\section{Metodología}

La metodología planteada para el desarrollo de la propuesta se realizó con base en los siguientes componentes.

- Emisiones de contaminantes por fuentes fijas en Bogotá 
- Análisis de la calidad de aire de Bogotá.

- Selección de los contaminantes objeto de cobro de tasa retributiva.

- Elementos técnicos y administrativos de la tasa.

- Estimación del valor de la tarifa para cada uno de los contaminantes objeto de cobro.

- Formulación del modelo de cálculo para determinar el monto total a pagar por concepto de tasa retributiva.

\section{Emisiones de contaminantes por fuentes fijas en Bogotá D. C.}

Bogotá por ser una de las ciudades más grandes del país y en su condición de capital de Colombia presenta un mayor crecimiento industrial; por consiguiente, altas cifras de contaminación atmosférica por la emisión de gases, vapores y humos aportados por fuentes fijas y móviles. La cuota de participación de emisiones de contaminantes atmosféricos por fuentes fijas en Bogotá, proviene generalmente de pequeñas, medianas y grandes industrias. El inventario de emisiones más reciente es el que presento la Universidad de los Andes en el 2004, en el sexto informe que hace referencia al inventario de emisiones para la ciudaá. En la Tabla 1, se presentan las emisiones atmosféricas estimadas para fuentes fijas en el perímetro urbano de Bogotá, en $\mathrm{Kg} /$ día y Ton/año. Las fuentes fijas aportan un $64 \%$ del total de las emisiones de $\mathrm{PM}_{10}$, un $68 \%$ de las emisiones de $\mathrm{SO}_{2}$, un $2 \%$ de $\mathrm{CO}$ y un $8 \%$ de las emisiones de NOx. De esta manera, se observa que los mayores aportes de emisiones de $\mathrm{PM}_{10}$, y $\mathrm{SO}_{2}$, provienen de las fuentes industriales.

Tabla 1. Emisiones Atmosféricas estimadas para Fuentes Fijas en Bogotá

\begin{tabular}{|c|c|c|}
\hline \hline CONTAMINANTE & Emisiones Kg/dнa & Emisiones Ton/aco \\
\hline $\mathrm{CO}$ & 21912 & 7998 \\
\hline $\mathrm{PM}_{10}$ & 8062 & 2943 \\
\hline $\mathrm{SO}_{2}$ & 14011 & 5114 \\
\hline $\mathrm{NO}_{\curlyvee}$ & 3825 & 1396 \\
\hline
\end{tabular}

Fuente: Inventario de emisiones Universidad de los Andes. 2004.

\section{Calidad del aire en Bogotá}

La Red de Monitoreo de Calidad del Aire de Bogotá (RMCAB) ha llevado un registro de las concentraciones de los contaminantes ( $P M_{10}, P S T, \mathrm{NO}_{x}, \mathrm{SO}_{2}, \mathrm{CO}, \mathrm{O}_{3}$, Benceno, Formaldehido, Tolueno) desde 1997. Está conformada por 13 estaciones automáticas de monitoreo de calidad del aire y 2 estaciones meteorológicas más, que detectan en forma continua la concentración de los principales contaminantes en la ciudad.

El análisis de las tendencias de la concentración de los contaminantes monitoreados por la RMCAB muestran que:

El $\mathrm{PM}_{10}$ es el contaminante con mayor índice de excedencias de la norma de calidad del aire, seguido por el ozono.

Las concentraciones de óxidos de nitrógeno, óxidos de azufre y monóxido de carbono, presentan pocas o ninguna excedencia de la norma. Las localidades de Puente Aranda, Kennedy y Fontibón, presenta las mayores.

Concentraciones de contaminantes, especialmente de $\mathrm{PM}_{10}$.

En la tabla 2 se presenta la comparación entre los valores sugeridos por la Organización Mundial de la Salud (OMS) - en su actualización de 2005- para contaminantes criterio, respecto con los valores establecidos en la Resolución 1208 de 2003 de la SDA.

A pesar de que la concentración de contaminantes en varias estaciones de la ciudad se encuentra por debajo de los niveles máximos permisibles definidos por la normatividad Nacional y Distrital, 
dicha concentración presenta valores muy elevados de los sugeridos por la OMS. Es por esto que se hace necesario implementar medidas complementarias a las de comando y control para asegurar el cumplimiento de las normas y garantizar un ambiente sano, reducir los riesgos a la salud humana y minimizar los impactos sobre el recurso.

Tabla 2. Comparación valores Resolución 1208 con valores de la Guía OMS.

\begin{tabular}{|c|c|c|c|}
\hline PARÁMETRO & $\begin{array}{c}\text { Límite Máximo per- } \\
\text { misible Res. 1208 mg/ } \\
\mathrm{m}^{3}\end{array}$ & $\begin{array}{c}\text { Guía OMS } \\
\text { actualización 2005 } \\
\mathrm{mg} / \mathrm{m}^{3}\end{array}$ & Tiempo de Exposición \\
\hline $\mathrm{PM}_{10}$ & 55 & 20 & Promedio Anual \\
\hline $\mathrm{SO}_{2}$ & 325 & 24 & Promedio 24 Horas \\
\hline $\mathrm{NO}_{2}$ & 100 & 40 & Promedio Anual \\
\hline $\mathrm{O}_{3}$ & 110 & 100 & Promedio en 8 horas \\
\hline $\mathrm{CO}$ & 11000 & 10000 & Promedio en 8 horas \\
\hline
\end{tabular}

Fuente Fuente Guía OMS

\section{Selección de contaminantes objeto de cobro}

Los contaminantes atmosféricos objetos de cobro se seleccionaron teniendo en cuenta los contaminantes que son monitoreados por la red de monitoreo de calidad de aire de Bogotá (RMCAB) y los contaminantes que son regulados por la normatividad ambiental Distrital por la resolución 1208 de 2003 tanto para fuentes de combustión externa como para fuentes fijas en procesos productivos. Se recomienda la reglamentación de la tasa retributiva para las emisiones de material particulado $\mathrm{PM}_{10}$, óxidos de azufre $\mathrm{SO}_{\mathrm{x}}$ y óxidos de nitrógeno $\mathrm{NO}_{\mathrm{x}}$.

\section{Elementos técnicos y administrativos de la tasa}

En la tabla 3 se describen los 4 elementos técnicos en los que debe centrarse la estructura de la tasa retributiva que son: el Hecho generador, la Base Imponible, el sujeto pasivo y activo.

Tabla 3. Elementos técnicos.

\begin{tabular}{||c|l||}
\hline \multicolumn{2}{|c|}{ ELEMENTOS TÉCNICOS } \\
\hline Hecho & $\begin{array}{l}\text { Constituye el hecho generador la utilización directa de la atmósfera, con el propósito } \\
\text { de descargar humos, vapores o gases y cuya acción genera consecuencias nocivas } \\
\text { sobre los recursos naturales. }\end{array}$ \\
\hline Sujeto Pasivo & $\begin{array}{l}\text { Son sujetos pasivos y estarán obligados al pago de la tasa retributiva las personas } \\
\text { naturales o jurídicas, de derecho público o privado, que utilicen directa o indirecta- } \\
\text { mente el recurso atmosférico urbano como receptor de la emisión de cualquiera de } \\
\text { los siguientes contaminantes: partículas suspendidas totales, óxidos de azufre y óxi- } \\
\text { dos de nitrógeno, aportados por fuentes fijas de combustión externa y/o fuentes fijas } \\
\text { de procesos productivos }\end{array}$ \\
\hline Sujeto Activo & $\begin{array}{l}\text { El sujeto activo de la tasa retributiva por emisión de contaminantes atmosféricos cor- } \\
\text { responde a la Secretaria Distrital de Ambiente de Bogotá como Autoridad Ambiental } \\
\text { del Distrito, quien es competente para cobrar y recaudar la tasa con base en los } \\
\text { reportes de emisiones atmosféricas presentadas por los sujetos pasivos }\end{array}$ \\
\hline Base Imponible & $\begin{array}{l}\text { La base imponible constituye la suma de las cantidades de cada uno de los contami- } \\
\text { nantes objetos de cobro de la tasa emitidos por el ducto o chimenea. Se definió el Ki- } \\
\text { logramo como unidad de carga contaminante emitida por las fuentes fijas puntuales. }\end{array}$ \\
\hline
\end{tabular}


La tabla 4 presenta los elementos administrativos de la tasa retributiva.

Tabla 4. Elementos Administrativos.

\begin{tabular}{|c|c|}
\hline \multicolumn{2}{|r|}{ ELEMENTOS ADMINISTRATIVOS } \\
\hline $\begin{array}{l}\text { Declaración } \\
\text { de emisiones }\end{array}$ & $\begin{array}{l}\text { Se presentarán anualmente ante la (SDA) un reporte de emisiones, de acu- } \\
\text { erdo con el formato diseñado para tal efecto. }\end{array}$ \\
\hline $\begin{array}{l}\text { Presentación de auto } \\
\text { declaraciones }\end{array}$ & $\begin{array}{l}\text { Se sugiere que la presentación de la autodeclaración y pago de la tasa re- } \\
\text { tributiva por parte de los sujetos pasivos, se realice según el Número de Iden- } \\
\text { tificación Tributaria, NIT, dentro de los plazos señalados. }\end{array}$ \\
\hline Comprobación & $\begin{array}{l}\text { El sujeto pasivo deberá tener a disposición de la SDA la Información en que } \\
\text { basa sus autodeclaraciones, para efectos de los procesos de verificación y } \\
\text { control que ésta realice o los procedimientos de reclamación que interponga. }\end{array}$ \\
\hline Forma de cobro & $\begin{array}{l}\text { La liquidación del monto total a pagar por concepto de tasa retributiva se } \\
\text { debe realizar en periodos anuales; este cobro se realizará mediante factura } \\
\text { expedida por la (SDA). }\end{array}$ \\
\hline
\end{tabular}

Fuente: elaboración propia

\section{Estimación de la tarifa}

Se formularon criterios técnicos que permitieron señalar el valor diferencial de la tarifa para cada uno de los contaminantes objeto de cobro, teniendo en cuenta los costos sociales que están relacionados con la inversión que realiza el Distrito para la atención a pacientes que presentan enfermedades respiratorias agudas ERA's asociadas a la contaminación atmosférica en Bogotá; y los costos del programa de control y vigilancia de la calidad del aire de Bogotá.

Se definieron las tarifas para el cobro de la tasa retributiva en $2810 \$ / \mathrm{Kg}$ para el $\mathrm{PM}_{10}, 2816 \$ / \mathrm{Kg}$ para los $\mathrm{SO}_{\mathrm{x}}$, y 2866 \$ Kg para los $\mathrm{NO}_{\mathrm{x}}$.

\section{Formulación del Modelo de Cálculo}

Se estableció el modelo de cobro de la tasa, el cual será el resultado de multiplicar la tarifa correspondiente para cada uno de los contaminantes objetos de cobro, expresada en $(\$ / \mathrm{Kg})$, por la carga contaminante emitida por la fuente expresada en ( $\mathrm{Kg} / \mathrm{día}$ ) por el número total de días de operación de la fuente de emisión al año.

La carga contaminante se calculara de conformidad con la siguiente formula:

$$
C C=\left\{\left[Q\left(\frac{m^{3}}{h}\right) * C\left(\frac{m g}{m^{3}}\right)\right] * 10^{-6} * \frac{t}{24}\left(\frac{h}{\text { dia }}\right)\right\}
$$

En donde,

Cc : Carga contaminante en kilogramos al día (kg/día).

Q : Caudal promedio en metros cúbicos hora.

C : Concentración de la sustancia contaminante en miligramos por metro cubico.

$10^{-6}$ : Factor de conversión de unidades.

t : Tiempo de funcionamiento de la fuente de emisión en horas al día.

El cálculo del monto anual a pagar por concepto de tasa retributiva se calculará de la siguiente forma. 
Donde:

$$
T R_{i}(\$)=\left[\begin{array}{c}
T_{i}\left(\frac{\$}{K g}\right) * C C_{i}\left(\frac{K g}{\text { dia }}\right) \\
* t(\text { dias })
\end{array}\right]
$$

$\mathrm{TR}_{i}=$ Tasa Retributiva por emisión del contaminante $i$.

$\mathrm{T}_{i}=$ Tarifa correspondiente al contaminante $i$.

$\mathrm{CC}_{i}=$ Carga Contaminante diaria de la sustancia $i$.

$\mathrm{t}=$ Días de funcionamiento de la fuente al año

El valor total a pagar por concepto de tasa retributiva por emisión de contaminantes atmosféricos será la sumatoria del cálculo de cada uno de los contaminantes emitidos por la fuente.

\section{Mecanismos de control y seguimiento por la autoridad ambiental}

\section{Autodeclaración de emisiones}

Se propone que los sujetos pasivos presenten anualmente ante la (SDA) un reporte de emisiones, de acuerdo con el formato que se ha diseñado para tal efecto. Estos reportes deben ser llevados a cabo por empresas consultoras registradas y autorizadas por el IDEAM para realizar evaluación de emisiones. Estas mediciones se deberán hacer bajo condiciones de operación normal de la fuente.

Los responsables de las fuentes fijas deberán efectuar como mínimo una evaluación de emisiones al año, salvo en los casos que ha consideración de la (SDA) se requieran más mediciones con el fin de determinar el impacto que causan estas emisiones. La Resolución 1208/2003 en sus artículos 13 y 19 a 30 establecen los métodos para la evaluación de emisiones en ductos y chimeneas y las recomendaciones que se deben seguir para efectos de reportar resultados confiables.

\section{Comprobación}

El sujeto pasivo deberá tener a disposición de la SDA la Información en que basa sus autodeclaraciones, para efectos de los procesos de verificación y control que esta realice o los procedimientos de reclamación que interponga el sujeto pasivo. La renuencia a las inspecciones dará lugar a la aplicación de las normas policivas establecidas en la normatividad ambiental.

\section{Forma de cobro}

Seproponequelaliquidación del montototal a pagarporconcepto de tasa retributivase realiceen periodos anuales, este cobro se realizará mediante factura expedida por la (SDA), la cual emitirá las respectivas facturas de conformidad con los requisitos exigidos en la Ley 633 de 2000 y en el Estatuto Tributario Nacional.

\section{Obligatoriedad de los límites permisibles}

El pago de las Tasas Retributivas no exonera a los usuarios del cumplimiento de los límites permisibles de las emisiones. Los límites permisibles de emisión de los contaminantes son los establecidos por la (SDA) en las respectivas normas de emisión de conformidad con la Resolución 1208 de 2003.

Tabla 5. Declaraciones

\begin{tabular}{|l|l|l|}
\hline Ultimo Digito NIT & Presentación Declaraciones & Fecha pago \\
\hline $0-4$ & Marzo-Abril & Julio \\
$5-9$ & Julio-Agosto & Noviembre \\
\hline
\end{tabular}




\section{Infracciones y Sanciones}

La autoridad ambiental ejercerá las funciones de policía que le atribuye la ley 99 de 1993 y en tal virtud podrá adoptar las medidas necesarias y utilizar los medios apropiados para asegurar el cumplimiento del pago de la tasa retributiva.

El recaudo de las tasas retributivas se realizará sin perjuicio de imposición de medidas preventivas y sancionatorias a que haya lugar, de conformidad con el Articulo 85 de la Ley 99 de 1993.

El sistema de tasas retributivas fue desarrollado con el propósito de controlar la contaminación de manera más eficiente que los instrumentos de comando y control; la aplicación de un instrumento económico como la tasa retributiva tiene sentido si a la par que se aplica el instrumento, se ofrecen incentivos que permitan al empresario mitigar y controlar sus emisiones motu propio.

\section{Incentivos}

Por lo anterior, se proponen los siguientes descuentos:

- Un descuento del $10 \%$ para aquellos usuarios que realicen pago anticipado. En la factura de cobro se establecerá la fecha para pago con descuento y la fecha límite de pago.

- Para aquellas empresas que se encuentren implementando o adelantando planes de reconversión a tecnologías limpias conforme lo establece el Decreto 948 de 1995, Artículo 100, parágrafo 2, se establecerá un descuento del $50 \%$ en el monto total liquidado de la tasa retributiva.

- La autoridad ambiental deberá realizar una auditoría a los industriales interesados en implementar los planes de reconversión a tecnologías limpias con el fin de establecer la validez de la reconversión tecnológica y la clasificación a la que aspira.

\section{Conclusiones}

La tasa retributiva por emisión de contaminantes atmosféricos se establece con el fin de cobrar a los responsables de las emisiones objeto de cobro, por el servicio que presta la atmosfera de recepción, asimilación y transporte de la carga contaminante, y al mismo tiempo por los daños que estas emisiones ocasionan a la salud humana y a los ecosistemas en general.

Considerando el impacto que causan las emisiones de contaminantes atmosféricos al deterioro de la calidad del aire, los efectos nocivos sobre la salud humana, y teniendo en cuenta los contaminantes que emiten las fuentes fijas y los contaminantes evaluados por la (RMCAB); se seleccionaron como contaminantes básicos para iniciar el cobro de la tasa retributiva el Material particulado, los Óxidos de Azufre y los Óxidos de Nitrógeno como los contaminantes atmosféricos objeto de cobro de la tasa retributiva en un primer desarrollo del programa.

El valor de la tarifa para los contaminantes objeto de cobro fueron expresadas en pesos por unidad de carga contaminante $(\$ / \mathrm{kg})$. El valor de la tarifa para el MP es de $2810 \$ / \mathrm{Kg}$, para el SO $2816 \$ / \mathrm{Kg}$ y para el NOx de $2866 \$ / \mathrm{Kg}$, estos valores se estimaron con base en los costos sociales relacionados a la inversión que realiza el Distrito Capital para la atención a pacientes que presentan enfermedades respiratorias agudas ERA's asociadas a la contaminación atmosférica en Bogotá; y los costos del programa de control y vigilancia de la calidad del aire de Bogotá.

Con base en el inventario de emisiones realizado por la Universidad de los Andes se encontró que las fuentes fijas puntuales corresponden a 4504 predios, de los cuales 106 contribuyen con más del $95 \%$ de la emisión industrial de todos los contaminantes sobre la ciudad. Las fuentes fijas aportan un $64 \%$ del total de las emisiones de MP, un $68 \%$ de las emisiones de $\mathrm{SO}_{2}$, un $2 \%$ de $\mathrm{CO}$ y un $8 \%$ de las emisiones de NOx.

Los instrumentos económicos más que instrumentos financieros para el control de la contaminación, son instrumentos que buscan cambiar el comportamiento del contaminador ya que incitan a la innovación tecnológica y el mejoramiento continuo del desempeño ambiental de las empresas favoreciendo la prevención de la contaminación. 
La poca información sobre la valoración económica de los impactos en salud relacionados con enfermedades causadas por la contaminación del aire, dificultó la estimación de un valor real de la tarifa aplicable a cada uno de los contaminantes atmosféricos; por esta razón, los valores presentados en esta propuesta se tratan de valores estimados que pueden no representar el valor real de la afectación a la salud por la acción de los contaminantes atmosféricos.

Se recomienda realizar estudios sobre la valoración económica de los impactos en la salud humana causados por enfermedades asociadas a la contaminación atmosférica en Bogotá con el fin de identificar, documentar y estimar daños y costos causados por la contaminación atmosférica, para que de esta manera se pueda obtener un valor real de los costos sociales que genera dicha contaminación.

Se recomienda realizar estudios sobre valoración económica de los daños que causa la contaminación atmosférica a los materiales, estructuras y monumentos de alto valor histórico-artístico con el fin de establecer costos que genera la contaminación a este tipo de bienes. Se recomienda que el valor de la tarifa este expresado en salario mínimo legal vigente (SMLV) de tal manera que cada año aumenta el valor de la tarifa según el aumento salarial establecido por el gobierno.

De igual manera, se recomienda que la autoridad ambiental en desarrollos posteriores del programa de tasas retributivas por emisión de contaminantes atmosféricos fije el cobro de tasa retributiva para contaminantes específicos como metales pesados y compuestos orgánicos volátiles (COV'S) donde las tarifas establecidas para estos contaminantes estén basadas en la toxicidad del contaminante.

Es necesario el fortalecimiento de la capacidad operativa, técnica y jurídica de la Secretaria Distrital de Ambiente, la cual debe contar con los recursos necesarios para medir, cobrar y recaudar continuamente la tasa retributiva para que así mismo este programa sea viable y efectivo. Se recomienda que los ingresos generados por el recaudo de la tasa retributiva deban ser destinados a programas encaminados a la protección y mejoramiento del recurso atmosférico.

\section{Referencias Bibliográficas}

[1] Arciniegas, A. \& Rodríguez C. (2005). Estudio de la morbilidad en niños menores a cinco años por Enfermedad Respiratoria Aguda y su relación con la concentración de partículas en la localidad de Puente Aranda. Bogotá. Trabajo de grado (Ingeniero Ambiental y Sanitario). Universidad de la Salle Facultad de Ingeniería Ambiental y Sanitaria.

[2] Bjorn, Larsen (2004). Cost of environmental damage: A Socio-Economic and Environmental Health Risk Assessment.

[3] Castro Hernández, L. F. (1997). Marco teórico conceptual para la implementación de las tasas retributivas. Ciudad: Corporación Autónoma.

[4] Centro de Investigaciones en Ingeniería Ambiental (2004). Diseño e implementación de un modelo de calidad de aire en Bogotá. Informe 6, Inventario de emisiones de fuentes fijas para Bogotá. Bogotá: Universidad de los Andes.

[5] Conpes (2005). Documento Conpes 3344; Lineamientos para la formulación de la política de prevención y control de la contaminación del aire. Bogotá, PN.

[6] Correa Restrepo, Francisco; De La Ossa Ar- teaga, Angélica; Vallejo Chanci, Zuly (2007). Regulación ambiental en Colombia: el caso de la tasa retri- butiva para el control de la contaminación hídrica. Semestre Económico, Vol.10 No.19. ISSN 0120-6346.

[7] Gaitán, M. et al.I. Análisis de la calidad del aire en Bogotá. 2007. Disponible en:http://revistaing. uniandes.edu.co/pdf/26a10.pdf?ri=565b5b2e5babb1ad326211f7e650b180.

[8] Garcia, Carolina \& Mosquera, Cesar (2008). Asociación entre la morbilidad por enfermedad respiratoria y contaminantes atmosféricos (MP, NO y O) en Fontibón y Puente Aranda de la ciudad de Bogotá. Trabajo de grado para optar al título de Ingeniero Ambiental y Sanitario de la Universidad de la Salle. 
[9] López, Tomás J. (2002) Fiscalidad ambiental: análisis y efectos distributivos. Granada. ISBN 9788484446163.

[10] _ _ (1995) Tasas por contaminación Hídrica. Consideraciones para su fijación. Mayo de 1995.

[11] Organización Mundial de la Salud (2005). Guías de calidad del aire relativas al material particulado, el ozono, el dióxido de nitrógeno y el dióxido de azufre. Actualización mundial 2005: ONU.

[12] Red de Monitoreo de Calidad de Aire de Bogotá (2004) Informe anual de calidad de aire de Bogotá, 2003.

[13] República de Colombia, (1974). Decreto ley 2811 de 1974. Bogotá: Imprenta Nacional. Ministerio de Salud, (1982). Decreto 02 de 1982. Bogotá: Imprenta Nacional.

[15] Ministerio del Medio Ambiente. (1996). Las tasas retributivas, el proceso de reglametación. Bogotá: Imprenta Nacional.

[16] Ministerio del Medio Ambiente. (1997). Política Nacional de Producción Más Limpia, República de Colombia, Santa Fe de Bogotá D. C.: Imprenta Nacional.

[17] Secretaria Distrital de Ambiente. (2003). Resolución 1208 del 2003. Por la cual se dictan normas sobre prevención y control de la contaminación atmosférica por fuentes fijas y protección del aire. DAMA. Bogotá: Imprenta Nacional.

[18] Secretaria Distrital de Salud (2004). "Lineamientos técnicos y administrativos para la prevención y atención de la Enfermedad Respiratoria Aguda (ERA). Bogotá, D. C.: Imprenta Nacional. 